\title{
Lithium and the Nature of Brown Dwarf Candidates
}

\author{
Rafael Rebolo ${ }^{1}$, Antonio Magazzù ${ }^{2}$, Eduardo L. Martín ${ }^{3}$ \\ ${ }^{1}$ Instituto de Astrofisica de Canarias, Via Lactea s/n, 38200 La Laguna, \\ Tenerife, Spain \\ ${ }^{2}$ Osservatorio Astrofisico di Catania, Città Universitaria, I-95125 \\ Catania,Italy \\ ${ }^{3}$ Astronomical Institute "Anton Pannekoek", Kruislaan 403, 1098 SJ, \\ Amsterdam, The Netherlands
}

\begin{abstract}
This paper discusses the ability of Li to confirm the substellar nature of a brown dwarf candidate. Theoretical computations using different interior models agree that brown dwarfs with masses below $\sim 0.065 \mathrm{M}_{\odot}$ preserve a significant fraction of their initial $\mathrm{Li}$ content while for higher masses total Li depletion occurs in very short timescales. Refined spectral synthesis using brown dwarf model atmospheres shows that the $\mathrm{Li}$ I resonance line at $670.8 \mathrm{~nm}$ should produce a conspicuous feature at effective temperatures higher than $2000 \mathrm{~K}$, suitable for spectroscopic detection in present brown dwarf candidates. We summarize the results of our search for $\mathrm{Li}$ in many of the best candidates known so far.
\end{abstract}

\section{Introduction}

The ${ }^{7} \mathrm{Li}$ isotope, the most abundant of the two stable lithium isotopes in Nature, has been detected in hundreds of stars and in the Interstellar Medium. The nuclear binding energy of ${ }^{7} \mathrm{Li}$ nuclei is rather low, as a consequence, these are efficiently destroyed in stellar interiors via proton collisions ${ }^{7} \mathrm{Li}(\mathrm{p}, \alpha)^{4} \mathrm{He}$ at relatively low temperatures, $\sim 2 \times 10^{6} \mathrm{~K}$. The behaviour of the cross-section for this reaction against the temperature of the plasma allows to define a ${ }^{7} \mathrm{Li}$ burning temperature such that at higher temperatures the stellar plasma is essentially $\mathrm{Li}$ free. This burning temperature has a weak dependence on the density conditions of the interiors, varying in the range 1.8-2.4 $\times 10^{6} \mathrm{~K}$ for objects close to the substellar limit. In this paper we deal with the ability of ${ }^{7} \mathrm{Li}$ nuclei, hereafter $\mathrm{Li}$, to allow distinguishing between very low mass stars and massive brown dwarfs (BD), not only on theoretical grounds, but also observationally through detection of Li features in the spectrum. Many of the ideas and results presented 
here can be found in Rebolo, Martín and Magazzù (1992), Magazzù, Martín and Rebolo (1993) and Martín, Rebolo and Magazzù (1994).

The present abundance of $\mathrm{Li}$ in our Galaxy is $\mathrm{Li} / \mathrm{H}=10^{-9}$, usually expressed as $\log N(\mathrm{Li})=3$ in the scale $\log N(\mathrm{H})=12$. Such present "cosmic" abundance is derived from observations of $\mathrm{T}$ Tauri stars, $\mathrm{F}$ stars in young stellar clusters like Pleiades and Hyades, and Interstellar Medium. On the other hand, observations of halo dwarfs show that the Li content in the material from which our Galaxy formed was at least $\log N(\mathrm{Li})=2$ (Spite \& Spite 1982, Rebolo et al. 1988). In fact, $\mathrm{Li}$ is one of the few nuclear species produced in the first minutes of the life of the Universe (Wagoner et al. 1967, Boesgaard \& Steigman 1985). Although there is still controversy on the exact abundance that came out from the Big Bang, it is possible to claim that any star or substellar object formed in our Galaxy was born with a lithium abundance higher than $\log N(\mathrm{Li})=2$. This is important in order to establish the existence of brown dwarfs.

\section{Lithium Destruction at the Bottom of the Main Sequence}

The fragility of Li nuclei in stellar interiors makes possible to use this element as a tracer of internal structure in stars of different type (see e.g. reviews by Michaud \& Charbonneau 1991, Rebolo 1991). Since about 30 years it is known that $\mathrm{Li}$ is destroyed very efficiently in the interior of solar type stars, but only recently it has been possible to estimate how efficient this destruction is in very late type stars. Observations of late K and M pre-main sequence (PMS) stars (Magazzù, Rebolo and Pavlenko 1992, Martín et al. 1994a) show that significant Li destruction takes place during the early evolution of PMS low mass stars. Early M-type stars in the Pleiades (age $70 \mathrm{Myr}$ ) have reduced their initial Li content more than a factor 1000 (García López, Rebolo and Martín 1994), even younger M3-M6 stars in the $\alpha$ Per open cluster (age $50 \mathrm{Myr}$ ) have destroyed Li by similar factors (Zapatero-Osorio et al., this volume). The absence of $\mathrm{Li}$ in $\alpha$ Per M5-M6 stars - masses in the range 0.2-0.1 $M \odot$ - is the strongest observational indication of the high efficiency of very low mass stars in destroying their initial Li content and is in good agreement with the theoretical predictions of D'Antona \& Mazzitelli (1994). As far as we know, the only very late M star retaining lithium in its atmosphere is UX Tau C (Magazzù et al. 1991), the faintest object of the UX Tau PMS system with mass lower than $0.2 M \odot$. This star presents a strong $\mathrm{Li}$ absorption resonance doublet, indicating that has suffered little destruction. However, the age uf the UX Tau system is only about 2 Myr! It can be inferred that very low mass stars with age greater than a few Myr have destroyed a large fraction of their initial Li. 


\section{The Lithium Test}

Visual inspection of the curves showing the evolution of central temperatures versus time for very low mass stars and brown dwarfs (see e.g. D'Antona and Mazzitelli 1985, Burrows and Liebert 1993) clearly denotes that objects with masses well below the substellar limit cannot reach the Li burning temperature. Opposite to very low mass stars, these brown dwarfs must preserve a significant amount of their initial $\mathrm{Li}$ content. The effective atmospheric temperatures of objects close to the substellar limit are subject of controversy and difficult to predict on theoretical grounds, however the available spectral type- $T_{\text {eff }}$ calibrations give effective temperatures of about $2500 \mathrm{~K}$ for the present brown dwarf candidates, not very different to those of the coolest $\mathrm{T}$ Tauri stars with detected Li. These considerations prompted Rebolo et al. (1992) to compute the formation of the Li I resonance doublet at $670.8 \mathrm{~nm}$ using Allard (1990) brown dwarf model atmospheres with $T_{\text {eff }} 2000$ to $2700 \mathrm{~K}$. The computations showed the formation of a very strong line (equivalent width of several $\AA$ ) in such cool atmospheres and moved us to suggest its detection as a powerful tool to confirm the substellar nature of brown dwarf candidates: the Li test (Rebolo et al. 1992)

We summarize in the following some recent results that support the applicability of the test. Both, regarding the destruction/preservation of Li around the substellar limit and the formation of Li lines in the atmospheres of very cool objects.

\subsection{The Mass Limit for Preservation of Lithium}

A simple approach considering the structure of a brown dwarf as a $n=3 / 2$ polytrope indicates that the central temperature of such an object never reaches the value necessary to burn lithium for $M \lesssim 0.07 M_{\odot}$. Calculations of Li depletion in substellar objects using detailed interior models show that Li is preserved below $0.06 M \odot$ (Magazzù et al. 1993). In fact, Pozio (1991), Stringfellow (1989) (quoted in Bessell and Stringfellow 1993) and Magazzú et al. had independently considered this problem and obtained similar results. More recently, Nelson et al. (1993) and D'Antona \& Mazzitelli (1994) find essentially the same, Li is preserved below $\sim 0.065 M \odot$. It is remarkable the good agreement between all these works despite the use of different interior models, opacities and screening factors. The mass limit for Li preservation is clearly below the substellar mass limit, usually accepted to lay between 0.08 and $0.07 \mathrm{M} \odot$.

In Fig. 1 we show the Li destruction curves from Magazzù et al. (1993). They illustrate, similarly to those obtained by other authors, an interesting additional point. For objects with masses $0.08-0.07 M \odot$ it is predicted total Li destruction, but this takes place only after $\sim 100 \mathrm{Myr}$ at $0.08 \mathrm{M} \odot$ and $\sim 200 \mathrm{Myr}$ at $0.07 \mathrm{M} \odot$. At the age of the Pleiades, these objects should also show Li in their atmospheres, the mass limit for Li preservation coincides here with the hydrogen burning mass limit. The shape of the destruction curves allow to predict a sharp transition between Li-poor and Li-rich objects at the bottom of the MS of a 
cluster like the Pleiades. Observations of fainter and fainter objects in young open clusters should lead to a sudden detection of Li at a given luminosity and spectral type, which in turn would define an empirical location of the substellar mass limit. In an older cluster like the Hyades (600 Myr) the luminosity of objects at the substellar limit would be slightly higher than that of objects preserving Li.

Fig. 1. Depletion of Li for masses close to the substellar limit, after Magazzù et al. 1993

We have seen above that all theoretical considerations indicate that lithium should be preserved below $\sim 0.065 M \odot$. There is indication that lithium - if present - can be detected spectroscopically at the physical conditions of the atmospheres of such objects.

\subsection{Detectability of Lithium in Brown Dwarfs.}

The formation of atomic and molecular Li lines in the spectrum of brown dwarfs is a problem requiring careful modelling. So far, we have investigated optical and infrared Li I lines using brown dwarf model atmospheres with $T_{\text {eff }}$ higher than $1800 \mathrm{~K}$. In a near future we plan to investigate the formation of lines from molecular species containing Li atoms. The computations by Rebolo et al. (1992) and Magazzù et al. (1993) clearly revealed the formation of strong Li lines at $670.8 \mathrm{~nm}$ in high gravity $(\log \mathrm{g}=5.0)$ low temperature $(2000 \mathrm{~K})$ models 
showing that at these temperatures the $\mathrm{Li}$ absorption is very strong and well detectable in the forest of molecular bands present in the spectrum. It was argued following Tsuji (1973) that the formation of molecules containing Li would be relevant at temperatures lower than $1500 \mathrm{~K}$ and that no significant effect on the formation of the resonance doublet were expected at higher $\mathrm{T}_{\text {eff }}$. Recently, Pavlenko et al. (1994) analyzed in detail the LTE and NLTE formation of Li I lines using Allard (1990) model atmospheres of $\mathrm{T}_{\text {eff }} 2000,2500$ and $3000 \mathrm{~K}$. In these new computations, the dissociation equilibria for seven Li molecules $(\mathrm{LiH}$, $\mathrm{LiO}, \mathrm{LiCl}, \mathrm{LiF}, \mathrm{LiBr}, \mathrm{LiI}, \mathrm{LiOH})$ was taken into account, and also considered atomic-molecular line lists available in the Li resonance line region. More than 20 atoms in two ionization states and 54 molecules were considered in the state equation system. The synthetic spectra were able to reproduce the TiO bands in the region around the $\mathrm{Li}$ resonance line, correctly describing positions and intensities of the observed bands. The resulting LTE computations show the formation of prominent Li I $670.8 \mathrm{~nm}$ lines (see Fig. 2), with equivalent widths of several $\AA$. The NLTE effects are found to be small, less than 0.1 dex in abundance. LTE and NLTE curves of growth for the weaker Li I lines at 610.3 and $812.6 \mathrm{~nm}$. are also given in Pavlenko et al. (1994).

Computations of the most promising IR Li I lines also confirm the resonance doublet as the most suitable feature for detecting atomic Li in brown dwarfs. Such detection is feasible for faint objects $(\mathrm{R} \sim 22)$ with the new generation of large 8-10 m telescopes using intermediate resolution spectroscopy (3-4 $\AA$ ).

\section{Searching for Li in Brown Dwarf Candidates}

The first Li search in BD candidates was performed by Magazzù et al. (1993). These authors observed a few relatively bright objects (GL 234 B, GL 473AB, GL 569B, etc) with high resolution spectroscopy (FWHM=0.4-0.8 $\AA$ ). Since, as explained above, the Li lines are expected to be very strong, Martín et al. (1994b) observed fainter objects (down to $\mathrm{V}=20.5$ ) with intermediate resolution (FWHM=2-4 $\AA$ ) using $4 \mathrm{~m}$ class telescopes at La Palma and La Silla observatories. The Keck $10 \mathrm{~m}$ telescope has allowed to reach similar faint magnitudes, but with a much higher resolution (FWHM $=0.2 \AA$, Marcy et al. 1994). None of these three works has been able to report a Li detection.

Brown dwarf candidates can be found in three different contexts; in binaries, in clusters, or free floating in the field. In the following, we discuss the Li nondetections in each of these contexts. 
Fig. 2. Spectral synthesis of the Li I resonance line (from Pavlenko et al. 1994) obtained using a brown dwarf model atmosphere of $T_{\text {eff }}=2500$ and $\log \mathrm{g}=5$. Line profiles for $\log$ $\mathrm{N}(\mathrm{Li})=3.0,2.0,1.0,0.0,-1.0$ and -2.0 are shown

\subsection{Very Low Mass Binaries}

The best way of determining a substellar mass is to obtain a complete orbital solution of a binary system containing a brown dwarf. However, this has not yet been possible, and presently we only have approximate solutions for a handful of systems. Given the uncertainties some known secondaries could have masses below the substellar limit. In Table 1 we summarize the Li searches in BD candidate secondaries. The upper limits to the LiI equivalent widths (EW) come from Magazzù et al. (1993) and Martín et al. (1994b), and have been corrected for the flux contribution of the primary. The lower limits to the masses derived from the Li non-detection come from comparison with the predictions by Nelson et al. (1993). The dynamical masses come from the compilation made by Burrows et al. (1989), and Ianna et al. (1988) for LHS 1047B. However, we note that the dynamical masses of GL 473AB are probably much larger (see Henry, this volume), and the error bar for LHS 1047B should be increased according to Henry \& McCarthy (1993). From the comparison of Li masses and dynamical masses in Table 1 we find that there is consistency within the error bars. None of these secondaries is a BD with mass less than about $0.065 M \odot$, but it is not ruled out that some could have masses very close to the substellar mass limit $(0.08$ 
$M \odot)$. Presently, neither the Li test, nor the precision of orbital parameters can discriminate between secondaries slightly above or below the substellar limit.

Table 1. Li searches in VLM binaries

\begin{tabular}{lllll}
\hline Object & Sp.T. & LiI $(\mathrm{m} \AA)$ & $\begin{array}{l}\mathrm{M} / \mathrm{M}_{\odot} \\
\text { Lithium }\end{array}$ & $\begin{array}{l}\mathrm{M} / \mathrm{M}_{\odot} \\
\text { Dynamical }\end{array}$ \\
\hline GL 65A,B & M6 & $<100$ & $>0.065$ & $(0.115,0.109) \pm 0.008$ \\
GL 234B & M6: & $<500$ & $>0.065$ & $0.08 \pm 0.01$ \\
GL 473AB & M6: & $<500$ & $>0.065$ & $(0.059,0.051) \pm 0.01$ \\
GL 623B & M6: & $<700$ & $>0.065$ & $0.114 \pm 0.042$ \\
LHS 1047B & M6.5: & $<500$ & $>0.066$ & $0.055 \pm 0.032$ \\
\hline
\end{tabular}

Note: the dynamical masses for GL 473AB and LHS 1047B are controversial (see text).

\subsection{Young Open Clusters}

Several surveys for BD in nearby open clusters have been carried out in the last few years (e.g. Jameson \& Skillen 1989, Simons \& Becklin 1992, Bryja et al. 1994). Dozens of BD candidates were identified on the basis of very red optical or IR colours, but for only very few of them do proper motion and spectroscopic studies exist. The Li test has been applied to very few of these candidates: the one discovered by Rebolo et al. (1992) in the $\alpha$ Per cluster, three of the PM candidates in the Pleiades (Hambly et al. 1993), and the faintest proper motion Hyades member in the sample of Bryja et al. (1994).

The Li test provides stronger mass constraints in $\alpha$ Per and the Pleiades than in the Hyades because of the difference in ages of about a factor ten. The substellar Li region (SLR), the region of the HR diagram where substellar objects preserving Li lay, has an upper mass limit of $0.08 M_{\odot}$ or higher for ages lower than about $10^{8} \mathrm{yr}$, while for ages of a few $10^{8} \mathrm{yr}$ the upper mass limit is $\sim 0.065 M_{\odot}$ (Martín et al. 1994b). Using the conversions from I-K colour to BC and $T_{\text {eff }}$ of Bessell (1991), all the BD candidates of Table 2 in $\alpha$ Per and the Pleiades fall inside the SLR, which is inconsistent with the large $\mathrm{Li}$ depletion observed. In order to bring the positions of these stars outside the SLR, they would have to be shifted by $200 \mathrm{~K}$ to hotter $\mathrm{T}_{\text {eff }}$. Current uncertainties in assigning temperatures to these objects could account for this shift.

The Li mass constraints (summarized in Table 2) imply that AP 0323, HHJ 3, 10 and 14 are not BDs. Fainter and redder objects than these have been detected towards the Pleiades (see Jameson in this volume), but their membership to the cluster needs to be confirmed before the decisive Li test is attempted. 
Table 2. Li searches in open cluster BD candidates

\begin{tabular}{llllll}
\hline Object & Sp.T. & LiI $(\mathrm{mA})$ & $\mathrm{M} / \mathrm{M}_{\odot}$ & Cluster & Ref. \\
\hline AP 0323+48 & \multirow{2}{*}{ M6 } & $<440$ & $>0.08$ & $\alpha$ Per & ZRMG \\
HHJ 3 & & $<190$ & $>0.08$ & Pleiades & MBG \\
HHJ 10 & M5.5 & $<300$ & $>0.08$ & Pleiades & MRM \\
HHJ 14 & & $<180$ & $>0.08$ & Pleiades & MBG \\
BHJ 358 & M6 & $<300$ & $>0.065$ & Hyades & MRM \\
& & & & & \\
\hline
\end{tabular}

References: MRM = Martín, Rebolo, Magazzù (1994); MBG = Marcy, Basri, Graham (1994); ZRMG = Zapatero-Osorio et al., this volume

\subsection{Isolated objects in the field}

The coolest objects where Li has been searched for are several high proper motion nearby field dwarfs (LHS 2397a, LHS 2243, LHS 2065, LHS 2924), with spectral types in the range M8-M9 V (Kirkpatrick, Henry \& Liebert 1993). The mass lower limit implied by the Li non-detections (Table 3 ) depends on the age assumed for these objects. Since they are not in clusters, their age is undetermined. If they are older than a few times $10^{8}$ yr their masses must be higher than $\sim 0.065 \mathrm{M}_{\odot}$, but if any of them happens to be younger than about $10^{8} \mathrm{yr}$ its mass would have to be above the substellar mass limit in order to account for Li depletion.

Table 3. Li searches in field BD candidates

\begin{tabular}{lllll}
\hline Object & Sp.T. & LiI $(\mathrm{mA})$ & $\mathrm{M} / \mathrm{M}_{\odot}$ & Ref. \\
\hline LHS 1070 & M5.5 & $<80$ & $>0.065$ & MBG \\
LHS 36 & M6 & $<80$ & $>0.065$ & MBG \\
CTI 1156+28 & M7 & $\leq 400$ & $\geq 0.065$ & MRM \\
VB 8 & M7 & $<200$ & $>0.065$ & MRM \\
LHS 248 & M7 & $<80$ & $>0.065$ & MBG \\
TVLM 868-110639 & M7.5 & $<500$ & $>0.065$ & MRM \\
TVLM 513-46546 & M8 & $\leq 300$ & $\geq 0.065$ & MRM \\
ESO 207-61 & M8 & $<300$ & $>0.065$ & MRM \\
LHS 2397a & M8 & $\leq 700$ & $\geq 0.065$ & MRM \\
LHS 2243 & M8 & $<50$ & $>0.065$ & MRM \\
GL 569B & M8.5 & $<500$ & $>0.065$ & MMR \\
LHS 2065 & M9 & $<150$ & $>0.065$ & MRM \\
LHS 2924 & M9 & $<250$ & $>0.065$ & MRM \\
\end{tabular}

References: MRM = Martín, Rebolo, Magazzù (1994); MBG = Marcy, Basri, Graham (1994); MMR = Magazzù, Martín, Rebolo (1993). 


\section{Concluding remarks}

Computations of Li destruction in objects close to the substellar limit and of the formation of Li lines using brown dwarf model atmospheres strongly support the use of the $\mathrm{Li}$ I resonance line at $670.8 \mathrm{~nm}$ to explore the substellar nature of brown dwarf candidates. Any object with mass below $\sim 0.065 \mathrm{M} \odot$ and effective temperature higher than $\sim 2000 \mathrm{~K}$ should present a prominent Li resonance line. At lower effective temperatures this line is expected to be still strong; no model atmospheres are currently available, though. The formation of these lines in brown dwarfs spectra as well as the possible depletion of Li into grains requires detailed investigation. Nevertheless, most of the available brown dwarf candidates have estimated temperatures that make suitable the observation of the Li $670.8 \mathrm{~nm}$ line. This feature has not been detected in any of the searches carried out so far in these objects, which is interpreted as a dramatic absence of $\mathrm{Li}$ in all of them. The mass constraints are more stringent for Pleiades objects than for those in binaries or in the field. It is not ruled out that some of the very cool field objects may be a brown dwarf, but its mass should be constrained in such case to the narrow range 0.065-0.08 $M \odot$. At present the Li test rules out a brown dwarf nature of the faintest proper-motion members in the Pleiades, but this cluster is very well suited for a future detection of Li just beyond the bottom of the Main Sequence.

\section{References}

Allard, F. (1990): Ph.D. Thesis, Univ. Heidelberg

Bessell, M.S. (1991): AJ, 101, 662

Boesgard, A.M., Steigman, G. (1985): ARAA, 23, 319

Bryja, C., Humphreys, R.M., Jones, T.J. (1994): AJ, 107, 246

Burrows, A., Hubbard, W.B., Lunine J.I. (1989): ApJ, 345, 939

Burrows, A., Hubbard, W.B., Saumon, D., Lunine, J.I. (1993): ApJ, 406, 158

Burrows, A., Liebert, J. (1993): Rev. Modern Physics, 65, 301

D'Antona, F., Mazzitelli, I. (1985): ApJ 296, 502

García López, R.J., Rebolo, R., Martín (1994): A\&A, 282, 518

D'Antona, F., Mazzitelli, I. (1994): ApJS, 90, 467

Henry, T.J., McCarthy, Jr., D.W. (1993): AJ, 106, 773

Ianna, P.A., Rohde, J.R., McCarthy, D.W. (1988): AJ, 95, 1226

Jameson, R.F., Skillen, I. (1989): MNRAS, 239, 247

Kirkpatrick, J.D, Henry, T.J., Liebert, J. (1993): ApJ, 406, 701

Kirkpatrick, J.D, Kelly, D.M., Rieke, G.H., Liebert, J., Allard, F., Wehrse, R. (1993): ApJ, 402, 643

Magazzù, A., Martín, E.L., Rebolo, R. (1991): A\&A 249, 149

Magazzù, A., Martín, E.L., Rebolo, R. (1993): ApJ, 404, L17

Magazzù, A., Rebolo, R., Pavlenko, Ya.V. (1992): ApJ, 392, 159

Martín, E.L., Magazzù, A., Rebolo, R. (1992): A\&A, 257, 186

Martín, E.L., Rebolo, R., Magazzù, A., Pavlenko, Ya.V. (1994a): A\&A, 282, 503

Martín, E.L., Rebolo, R., Magazzù, A. (1994b): ApJ, Nov 20 
Marcy, G.W., Basri, G., Graham, J.R. (1994): ApJ, 428, L57

Michaud, G. Charbonneau, P. (1991): Space Sci Rev. 57, 1

Nelson, L.A., Rappaport, S., Chiang, E. (1993): ApJ, 413, 364

Pavlenko, Ya.V., Rebolo, R., Martín, E.L., García López (1995): A\&A, submitted

Pozio, F. (1991): Mem. Soc. Astr. Ital., 62, 171

Rebolo, R. 1991, "Evolution of Stars: The Photospheric Abundance Connection", eds G. Michaud and A. Tutukov, IAU Symp. 145, Kluwer, 85.

Rebolo, R., Martín, E.L., Magazzù, A. (1992): ApJ, 389, L83

Rebolo, R., Molaro, P., Beckman, J.E. (1988) A\&A, 192, 192

Simons, D.A., Becklin, E.E. (1992): ApJ, 390, 431

Spite, F., Spite, M. (1982): A\&A, 115, 357

Stringfellow, G.S. (1989): PhD Thesis. Univ. Calif. Santa Cruz

Tinney, C.G., Mould, J.R., Reid, I.N. (1993): AJ, 105, 1045

Tsuji, T. (1973): A\&A, 23, 411

Wagoner, R.V., Fowler, W.A., Hoyle,F. (1967): ApJ, 148, 3

This book was processed by the author using the $\mathrm{T}_{\mathrm{E}} \mathrm{X}$ macro package from SpringerVerlag. 